\title{
BMJ Open Brighter children? The association between seasonality of birth and child IQ in a population-based birth cohort
}

\author{
Nina H Grootendorst-van Mil, ${ }^{1,2,3}$ Régine P M Steegers-Theunissen,, 3 \\ Albert Hofman, ${ }^{5}$ Vincent W V Jaddoe, ${ }^{1,4,5}$ Frank C Verhulst, ${ }^{2}$ Henning Tiemeier ${ }^{2,5,6}$
}

To cite: Grootendorst-van Mil NH, Steegers-

Theunissen RPM, Hofman A, et al. Brighter children? The association between seasonality of birth and child IQ in a population-based birth cohort. BMJ Open 2017:7:e012406

doi:10.1136/bmjopen-2016012406

- Prepublication history and additional material is available. To view please visit the journal (http://dx.doi.org/ 10.1136/bmjopen-2016012406).

Received 27 April 2016 Revised 19 October 2016 Accepted 17 November 2016

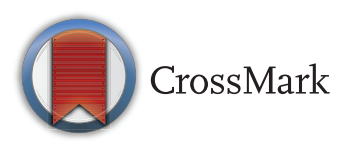

For numbered affiliations see end of article.

Correspondence to Dr Henning Tiemeier; h.tiemeier@erasmusmc.nl

\section{ABSTRACT}

Objective: Season of birth has repeatedly been found to be a risk indicator for adverse neurodevelopmental outcomes. Several explanations for this finding have been put forward but no conclusion has been reached. In the current study, we explored the role of sociodemographic and biological factors in the association between season of birth and child $\mathrm{IQ}$.

Design: In a prenatally recruited birth cohort (born in 2002-2006), we examined the association between season of birth and non-verbal IQ at age 6 years among 6034 children. We explored how adjusting for socioeconomic status and maternal $I Q$, childbirth outcomes, pregnancy vitamin $D$ status, nutritional intake, exposure to infections, and child age relative to peers in class changed the relation between season of birth and child $I Q$.

Results: We found that spring birth was associated with lower non-verbal IQ (estimate: more than 1 point; $\beta-1.24(95 \% \mathrm{Cl}-2.31$ to -0.17$), p=0.02$; seasonal trend $\beta-0.40(95 \% \mathrm{Cl}-0.74$ to -0.07$), p=0.02)$ than birth in summer. Adjustment for different covariates led to a substantial reduction ( $-65.0 \%$ change, in a seasonal trend analysis) of this association. In particular, sociodemographic factors and maternal IQ $(-10.0 \%$ and $-22.5 \%$ change, respectively) contributed.

Conclusions: Season of birth is an indicator of many underlying factors related to child IQ. The observed effects on IQ were small and therefore not of clinical significance.

\section{INTRODUCTION}

Season of birth has repeatedly been studied as a risk indicator for adverse neurodevelopmental outcomes. Consistently a winter-spring excess of births in cases of schizophrenia, bipolar disorder and schizoaffective disorder as well as major depression and autism have been described. ${ }^{1}$ Season of birth showed a similar association with cognition. ${ }^{2}$ The recurring question is: What hides behind the season of birth? Many different explanations for this relationship have been put forward but studies remain inconclusive.

\section{Strengths and limitations of this study}

- Population-based study population with a large sample size and prospective data assessments.

- This study took into account multiple seasonally patterned biological exposures during pregnancy to shed light on the suggested association with health outcome in the literature.

- Information on several prenatal factors was available from parental-reported questionnaires or single laboratory measurements only which could have led to misclassification.

- It is questionable how the observed difference in childhood translates into performance in everyday life.

Mostly it is speculated that seasonally patterned biological exposures during pregnancy explain the variation in health outcome by season of birth. However, an integral test of these possible explanations for the risk of poor neurodevelopmental outcomes is lacking and newer hypotheses, such as variation in vitamin $\mathrm{D}$ levels, have not been investigated.

Exposure to viral infections during pregnancy has been most extensively studied. The majority of studies focused on maternal influenza as the primary cause for a higher prevalence of schizophrenia associated with certain birth months. ${ }^{3}$ Poor prenatal nutrition has been proposed to be related to the birth seasonality of individuals who develop schizophrenia. ${ }^{4}$

Adverse birth outcomes are risk factors for impaired cognitive development. ${ }^{5}$ Previous studies have repeatedly described relationships between season of birth and birth weight, and duration of gestation. ${ }^{7}$

Recently, a strong interest in the effects of vitamin $\mathrm{D}$ on development of the brain emerged. Owing to its relationship with the duration of the photoperiod, low vitamin D levels are put forward as a potential 
mechanism between season of birth and neurological outcomes. $^{8}$

Another candidate mechanism for the seasonal patterns in intellectual ability are the educational policies regarding school entry. ${ }^{9}$ Age at school entrance and the relative age compared with peers are considered to affect the youngest in class. Adjustment for age at IQ test removes the impact of age on test scores. ${ }^{10}$ However, children relatively younger to peers in class (ie, those born in summer) will have received relatively more formal schooling at any given age than classmates born in other seasons.

The seasonal distribution of births within countries exhibits large fluctuations that remain remarkably constant over time. In the Netherlands, birth rates are highest in August-September and lowest in December and May (see online supplementary figure S1). Most probably, sociodemographic and sociocultural factors influence the amplitude of monthly birth rates, ${ }^{11}$ suggesting that overall births are not completely randomly distributed over the calendar year.

Against this background, we tested factors hypothesised to underlie the association of birth season with child cognitive development. In a large populationbased study, we first examined the association between season of birth and non-verbal IQ at age 6 years ((1) basic model), adjusted for child gender and age at sitting the IQ test. Next, we determined in additional models whether this association could be explained by specific variables-thereby testing different hypotheses; (2) sociodemographic factors and maternal IQ; (3) child birth weight and gestational duration; (4) pregnancy vitamin D plasma status; (5) maternal nutritional intake; (6) maternal exposure to fever during pregnancy; and (7) child age relative to peers at school. The magnitude of the effect estimate change due to these variables was calculated.

\section{METHODS}

Design and study population

This study was embedded in the Generation R Study, an ongoing population-based birth cohort from fetal life onwards in Rotterdam, the Netherlands. The Generation R Study, designed to identify early environmental and genetic determinants of growth, development and health, has been previously described in detail. ${ }^{12}$ In short, children were born between April 2002 and January 2006 to mothers who were enrolled in pregnancy $(n=8880)$ or at birth $(n=1017$; total $\mathrm{n}=9897$ ). Data collection in mothers, fathers and preschool children included questionnaires, detailed physical and ultrasound examinations, behavioural observations and biological samples. In total, 8305 children were participating in the postnatal phase at school age. For this study, we selected all mother-child dyads with available data on child IQ assessed at 6 years of age $(\mathrm{n}=6034 ; 73 \%)$.
Possible factors suggested to explain the association between seasonality of birth and IQ were categorised into hypotheses-based groups of variables as described above.

\section{Assessment of child period of birth and age}

Information on birth date was derived from the medical records completed by midwives and gynaecologists. These dates were used to define four astronomical seasons in the Netherlands: spring (March 21 to June 20), summer (June 21 to September 20), fall (September 21 to December 20) and winter (December 21 to March 20). We also tested meteorological seasons. This approach resulted in comparable associations with child $\mathrm{IQ}$ but data were not used for further analyses.

To calculate the relative child age compared with peers at school, we compared date of birth with the chronological distance from 30 September, the cut-off date in the Netherlands that determines enrolment in a class. ${ }^{13}$ We computed the number of days that a student was older than the theoretical youngest student in the class as we had no directly assessed data on the average age of peers in class of the children included in this study.

\section{Assessment of maternal and child IQ}

Around their sixth birthday (mean age $=6.0 \pm 0.3$ years), the children were invited to visit the Generation $R$ research centre. During this visit, children's non-verbal IQ was assessed using two subtests of the Snijders-Oomen Niet-verbale intelligentie Test-Revisie (SON-R 21/2-7)..$^{14}$

The test subsets used were mosaics (assesses spatial visualisation abilities) and categories (assesses abstract reasoning abilities). Raw scores were derived for each subtest and standardised to reflect a mean and SD of the Dutch norm population age $21 / 2-7$ years. The sum of the standardised scores of the two subtests were converted into the SON-R IQ score using age-specific reference scores provided in the SON-R $2^{1 / 2} 7$ manual (mean $=100, \mathrm{SD}=15$ ). The use of the subsets is warranted as the correlation between the IQ scores based on the two subtests and the full SON-R IQ battery was high $(\mathrm{r}=0.86$, Tellegen, personal communication).

The average reliability of the SON-R $2^{1 / 2} 7$ IQ score is 0.90 , range $0.86-0.92$ for the respective age. ${ }^{14}$ The reliability of the subtests that were used in our study are: 0.73 for mosaics and 0.71 for categories.

In the Generation R Study, $54.4 \%$ of the children are of non-Dutch origin. Owing to differences in exposure to the Dutch language in young children of different national origin, we have chosen to measure non-verbal intelligence.

During the child's visit to the research centre, maternal non-verbal IQ was assessed using a computerised version of the Ravens Advanced Progressive Matrices Test, set $\mathrm{I}^{15}$ Detailed information about these tests has been described. ${ }^{16}$ 


\section{Sociodemographic characteristics}

Information on maternal age, parity, educational level and national origin was obtained from questionnaires at enrolment. Maternal national origin was based on country of birth of the mothers' parents (Dutch, Cape Verdean, Moroccan, Turkish, Surinamese, Antillean and other national origin) and classified in accordance with Statistics Netherlands. Educational level was categorised into primary, secondary and higher education. Parity was classified in two categories: nulliparous and multiparous.

\section{Assessment of birth weight and gestational duration}

To estimate gestational age, crown-rump length (until a gestational age of 12 weeks and 5 days) or biparietal diameter (from 12 weeks and 5 days onwards), measured by fetal ultrasound examination, as previously described, were used. Interobserver and intraobserver intraclass correlation coefficients were all $>0.98$. Information on birth weight of the child was obtained from community midwifery and hospital registries. Birth weight was established directly postpartum and expressed in kilograms $(\mathrm{kg})$. Birth weight was expressed as SD score. These SD scores were constructed based on distributions in the Generation R cohort. ${ }^{17}$

\section{Assessment of vitamin D status in maternal mid-pregnancy and cord blood plasma samples}

Total vitamin $\mathrm{D}$ was reported as the sum of 25-hydroxyvitamin D2 (25OHD2) and 25-hydroxyvitamin D3 (25OHD3) species measured in maternal plasma and cord blood samples using a modification of a method previously described.$^{18}$ In mid-pregnancy (mean 20.6 weeks; SD 1.0, IQR 1.14), venous blood samples were collected and directly after delivery (mean gestational duration 39.8 weeks; SD 1.8, IQR 2.0), midwives or obstetricians collected cord blood from the umbilical vein. All samples were stored at $-80^{\circ} \mathrm{C} .{ }^{19}$ Vitamin D concentrations were measured in EDTA plasma samples at the Queensland Brain Institute from July 2013 to August 2014. Samples were quantified using isotope dilution liquid chromatography-tandem mass spectrometry. The analytical system comprised a Shimadzu Nexera ultra performance liquid chromatography (UPLC) coupled to an AbSciex 5500 QTRAP equipped with an atmospheric pressure chemical ionisation (APCI) source. Chromatographic separation was achieved using a Kinetex XB-C18 column $(50 \times 2.1 \mathrm{~mm}, \quad 1.7 \mu \mathrm{m}$; Phenomenex, USA) and $72 \%$ acetonitrile $/ 32 \%$ aqueous $0.1 \%$ formic acid at a flow rate of $0.5 \mathrm{~mL} / \mathrm{min}$. Interassay inaccuracy and imprecision were assessed at four concentration levels for 25OHD3 (48.3, 49.4, 76.4, $139.2 \mathrm{nmol} / \mathrm{L})$ and a single level $(32.3 \mathrm{nmol} / \mathrm{L})$ for 25OHD2 using certified reference materials purchased from the National Institute of Standards and Technology (NIST standard reference materials 972a levels 1-4), and were excellent at all concentration levels tested $(<10 \%$ and $<17 \%$, respectively). Assay repeatability was assessed via replicate analysis of an independent reference material (NIST SRM1950, $61.9 \mathrm{nmol} / \mathrm{L} 25 \mathrm{OHD} 3$ ), with interassay inaccuracy and imprecision $<11 \%(\mathrm{n}=343)$.

\section{Assessment of maternal pregnancy nutritional intake and plasma folate concentration}

In early pregnancy, the nutritional intake of the previous 3 months was assessed by using a modified version of a validated semiquantitative food frequency questionnaire (FFQ). ${ }^{20}$ The FFQ consists of 293 food items and is structured according to meal patterns. Questions in the FFQ include consumption frequency, portion size, preparation method and additions of the foods. Portion sizes were estimated using household measures and photographs. ${ }^{21}$ To calculate average daily nutritional values, the Dutch food composition table 2006 was used. ${ }^{22}$

On the basis of their seasonal variations in previous literature, we selected fat, carbohydrate, vitamin C, B, sodium, potassium, calcium, phosphorus and zinc intakes and adjusted for energy intake. ${ }^{23}$

Folate concentrations were analysed in EDTA plasma samples, drawn in early pregnancy (median 13.1 weeks of gestation; $90 \%$ range 10.5-16.9 weeks). Samples were analyses at the Department of Clinical Chemistry of the Erasmus Medical Center, Rotterdam, in 2008 using an immunoelectrochemiluminescence assay on the Architect System (Abbott Diagnostics BV). The between-run coefficient of variation depending on folate concentration varied between $1.5 \%$ and $8.9 \%$. Analytic ranges for folate concentrations were $1.8-45.3 \mathrm{nmol} / \mathrm{L}^{19}$

\section{Maternal exposure to infection during pregnancy}

Maternal C reactive protein (CRP) during midpregnancy was measured in EDTA plasma samples at the Department of Clinical Chemistry of the Erasmus Medical Center in 2009 using an immunoturbidimetric assay on the Architect System (Abbot Diagnostics BV, Hoofddorp, the Netherlands). The within-run precision for CRP was $1.3 \%$ at $12.9 \mathrm{mg} / \mathrm{L}$ and $1.2 \%$ at $39.9 \mathrm{mg} / \mathrm{L}$. The lowest concentration of detection was $0.2 \mathrm{mg} / \mathrm{L}$.

Information on maternal fever during the previous 3 months was collected from three prenatal questionnaires during early, mid-pregnancy and late pregnancy and coded as yes or no.

\section{Statistical analyses}

We used linear regression models to explore seasonal trends and covariate effects on child non-verbal IQ at age 6 years. In all analyses, non-verbal IQ was the dependent continuous variable.

First, bivariate correlation coefficients for predictor and outcome variables were calculated using Pearson correlation test. Second, we studied the association between child season of birth and non-verbal IQ. We examined whether different seasons of birth, defined in four categories, were related to IQ. We also tested the presence of a sinusoidal pattern in this relation. A sinusoidal curve was fitted to the data by introducing one sine and one cosine function in a regression model with 
a period of 6 months. An F test was used to test the joint effect of the sine and cosine functions.

In the third step, we tested the explanatory value of factors related to the seven formulated hypotheses. We added these variables both separate and step by step to the models. We first examined the association between child season of birth and non-verbal IQ and adjusted for child gender and age at sitting the IQ test ((1) basic model). Next, the following variables were added stepwise to this basic model; (2) sociodemographic factors and maternal IQ; (3) child birth weight and gestational duration; (4) maternal mid-pregnancy and cord blood vitamin D plasma concentrations; (5) maternal nutritional intake; (6) maternal exposure to fever during pregnancy; and (7) child relative age in class. In all analyses, we considered the change in $\mathrm{R}^{2}$ to evaluate the effect estimate change.

If plasma measurements were used as a determinant, we additionally adjusted for gestational duration at blood sampling.

The covariates can be separated in two categories; first, sociodemographic and maternal characteristics that may affect the timing of conception (1) and second, variables that are affected by the season in which the women is pregnant (2). The first can be considered as common causes of season of birth and child IQ while the latter are intermediate factors on the causal pathway between season of birth and child IQ. We have depicted these different models in diagrams (see online supplementary figure S2). We first test common causes and then test intermediate factors because the first are also potential confounders of intermediate pathways.

Missing data on the covariates were imputed using the Markov Chain Monte Carlo multiple imputation technique. Data on exposure (season of birth) and outcome (non-verbal IQ) were not imputed. Ten data sets were created and on each completed data set, multivariable regression analyses were performed. Afterwards, results were combined to one pooled estimate and for all analyses results including imputed missing data are presented. The percentages of missing values within the population for analysis were lower than $20 \%$, except for maternal pregnancy nutritional intake (29.9\%), presence of maternal fever during pregnancy $(21.5 \%)$, maternal pregnancy CRP concentrations $(34.9 \%)$, and maternal mid-pregnancy and cord blood vitamin D plasma concentrations $(23.8 \%$ and $45.9 \%)$. All analyses and data imputations were performed using SPSS software, V.21.0 (IBM Corp, Armonk, New York, USA).

\section{RESULTS}

Of all children who enrolled in the Generation $\mathrm{R}$ cohort before or at birth, $70.5 \%$ participated in the follow-up measurements at the age of 6 years; $16.5 \%$ of the mother-child pairs provided consent for further follow-up but did not visit the research centre, $10.0 \%$ were lost to follow-up, and $3.1 \%$ did not provide consent for further follow-up from the age of 6 years onwards.

\begin{tabular}{|c|c|c|c|c|}
\hline$(n=6034)$ & Spring & Summer & Autumn & Winter \\
\hline \multicolumn{5}{|l|}{ Maternal characteristics } \\
\hline Age, years & $30.7(5.1)$ & $30.3(5.2)$ & $30.7(5.0)$ & $30.6(5.2)$ \\
\hline \multicolumn{5}{|l|}{ National origin (\%) } \\
\hline Dutch & 52.3 & 53.0 & 55.3 & 54.3 \\
\hline Other Western & 12.4 & 10.9 & 11.1 & 11.2 \\
\hline Non-Western & 35.3 & 36.1 & 33.5 & 34.5 \\
\hline Parity (\% primiparous) & 51.4 & 55.0 & 57.3 & 53.6 \\
\hline \multicolumn{5}{|l|}{ Educational level (\%) } \\
\hline Primary & 10.9 & 10.0 & 4.8 & 10.1 \\
\hline Secondary & 39.5 & 40.3 & 39.5 & 41.3 \\
\hline High & 49.6 & 49.7 & 49.7 & 48.6 \\
\hline \multicolumn{5}{|l|}{ Smoking during pregnancy (\%) } \\
\hline Never & 78.4 & 77.2 & 75.4 & 75.6 \\
\hline Until pregnancy was recognised & 7.1 & 8.1 & 7.1 & 9.0 \\
\hline Continued & 14.5 & 14.6 & 17.4 & 15.2 \\
\hline \multicolumn{5}{|l|}{ Mode of delivery (\%) } \\
\hline Spontaneous & 69.1 & 70.3 & 67.8 & 72.5 \\
\hline Instrumental & 17.5 & 16.0 & 18.9 & 16.4 \\
\hline Caesarean section & 13.4 & 13.7 & 13.3 & 11.1 \\
\hline \multicolumn{5}{|l|}{ Child characteristics } \\
\hline Gender (\% boys) & 51.4 & 49.0 & 51.0 & 51.8 \\
\hline Birth weight, $\mathrm{kg}$ & $3.4(5.7)$ & $3.4(5.8)$ & $3.4(5.5)$ & $3.4(5.8)$ \\
\hline Gestational age at birth, weeks (median, IQR) & $40.0(1.9)$ & $40.1(2.0)$ & $40.1(2.0)$ & $40.0(2.0)$ \\
\hline
\end{tabular}


The general characteristics of the children and their mothers are presented in table 1 . In total, 1.441 $(23.9 \%)$ children were born in spring, $1.617(26.8 \%)$ in summer, $1.652(27.4 \%)$ in autumn and $1.324(21.9 \%)$ in winter.

We found no statistical evidence for a sinusoidal pattern of child non-verbal IQ at age 6 years by month of birth (adjusted for child gender and age at IQ test sine function $\beta-0.39$ (95\% CI -0.93 to 0.15 ), $\mathrm{p}=0.16$, cosine function $\beta-0.81$ (95\% CI -0.76 to 0.32$), \mathrm{p}=0.42$, joint sine-cosine function $\mathrm{F}=1.34$, $\mathrm{p}=0.26$ ).

A birth date in spring was associated with a more than one point lower non-verbal IQ score at age 6 years than birth in summer (adjusted for child gender and age at IQ test $\beta-1.24$ (95\% CI -2.31 to -0.17$), \mathrm{p}=0.02$; seasonal trend $\beta-0.40$ (95\% CI -0.74 to -0.07$), \mathrm{p}=0.02$ ).

As shown in the correlation matrix (see online supplementary table S1), child spring birth negatively correlated with nulliparity $(\mathrm{r}=-0.03, \mathrm{p}=0.008)$, maternal mid-pregnancy and cord blood vitamin $\mathrm{D}$ plasma concentrations $(\mathrm{r}=-0.11, \mathrm{p}=0.01$ and $\mathrm{r}=-0.12, \mathrm{p}<0.001)$, child relative age compared with peers in school $(\mathrm{r}=$ $-0.17, \mathrm{p}<0.001)$ and positively correlated with maternal CRP concentrations $(\mathrm{r}=0.05, \mathrm{p}=0.001)$ and presence of fever in mid-pregnancy $(\mathrm{r}=0.06, \mathrm{p}=0.001)$.

Next, we tested the change of the association between season of birth and IQ with addition of possible covariates to the basic model, each in a separate model (see online supplementary table S2). The maternal sociodemographic factors explained $10.3 \%\left(\mathrm{R}^{2}\right.$ change 0.088 , $\mathrm{p}<0.001)$ of the association between child season of birth and IQ and maternal IQ explained $39.3 \%\left(\mathrm{R}^{2}\right.$ change $0.085, \mathrm{p}<0.001)$. Maternal nutritional intake, child relative age in class and season at IQ assessment, gestational duration and birth weight and maternal infections during pregnancy explained, respectively, $11.2 \% ; 1.1 \%$, $6.2 \%$ and $8.3 \%$ of this association $\left(\mathrm{R}^{2}\right.$ change 0.034 , $\mathrm{p}<0.001 ; \mathrm{R}^{2}$ change 0.001, $\mathrm{p}=0.03 ; \mathrm{R}^{2}$ change 0.019, $\mathrm{p}<0.001$ and $0.002, \mathrm{p}=0.34$ ). Maternal plasma and cord blood vitamin D plasma levels explained $124.8 \%$ of the variance in this basic model not adjusted for potential confounders $\left(\mathrm{R}^{2}\right.$ change $\left.0.050, \mathrm{p}<0.001\right)$.

When we entered the covariates step by step (table 2) to the child's age-adjusted and gender-adjusted model, sociodemographic characteristics and maternal IQ substantially changed the association of child season of birth and IQ $\left(\mathrm{R}^{2}\right.$ change 0.088 and $0.036, \mathrm{p}<0.001$, respectively). Gestational duration and birth weight resulted in a modest improvement of the model $\left(R^{2}\right.$ change 0.010 , $\mathrm{p}<0.001)$. The further improvement of the model by other factors was marginal $\left(\mathrm{R}^{2}\right.$ change $\left.<0.002\right)$.

Together, adjustment for all factors examined resulted in a $65 \%$ reduction (total $\mathrm{R} 15.9 \%, \mathrm{~F}: \mathrm{p}<0.001$ ) of the association of child season of birth and non-verbal IQ at age 6 years (table 2).

Owing to the relatively large variance explained and the relatively large correlation with child IQ, we further explored the association between maternal IQ and child
S-

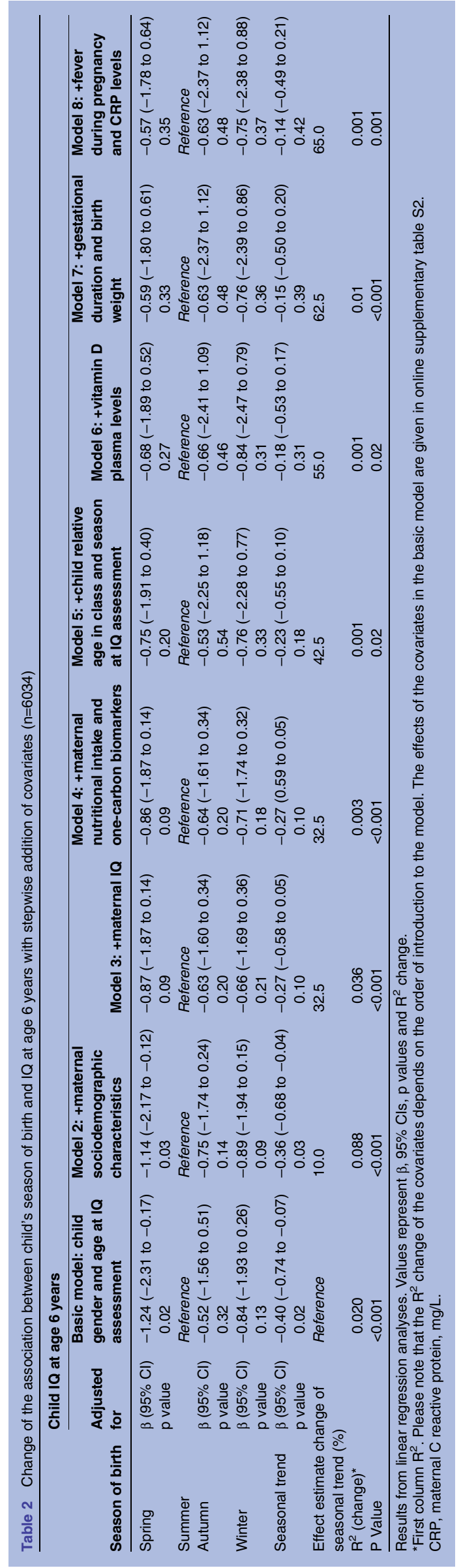


eason of birth. Mothers who gave birth in spring also scored -1.24 IQ points ( $\beta(95 \%$ CI -2.34 to -1.42$)$, $\mathrm{p}=0.03$ ) lower as compared with mothers who delivered in summer.

In all previous analyses, data based on imputations of covariates were used. To test robustness of results, we present the results of complete cases analysis $(n=1394)$ on the association between child season of birth and IQ. This showed results comparable to analyses based on imputed data: basic model $\beta-1.38,95 \%$ CI -3.52 to 0.75 , $\mathrm{p}=0.02$; model 3 , adjusted for maternal sociodemographic characteristics and maternal IQ $\beta-1.28,95 \%$ CI -3.31 to $0.76, \mathrm{p}=0.22$; and model 8 , adjusted for all included covariates $\beta-0.21,95 \% \mathrm{CI}-2.64$ to $3.04, \mathrm{p}=0.86$.

\section{DISCUSSION}

In this study, season of birth was a risk indicator for child IQ; spring-born children had a 1.2-point lower non-verbal IQ scores than children born in summer. Several different mechanisms partly account for this association; if anything, the effect of maternal sociodemographic factors and $I Q$ was most prominent.

Research on preferences of season to give birth is relatively scarce. Between 1991 and 1993, Basso $e t a l^{24}$ interviewed nearly 5000 women in the fertile age range in five countries across Europe. In this study, summer was the preferred time to begin a pregnancy in most countries, which would lead to birth in spring if conception occurs. In the Netherlands, the observed peak in live births has shifted from spring to late summer since the 1970s. This corresponds to the increase in use of oral contraceptives. ${ }^{25}$ In previous literature, the distinctness of a seasonal pattern varied by socioeconomic status. Married women and higher educated women showed a much stronger seasonal pattern of childbirth than unmarried and low education women. One could argue that these women were better able to plan their conception in the preferred period. ${ }^{26}$

Our study suggests that parental characteristics can partly account for the association between seasonality of birth and child intelligence. Buckles and Hungerman ${ }^{12}$ reported in their study of 52000 live births born between 1989 and 2001 in the USA that mothers delivering in winter were in general more often teenagers, unmarried and lacked a high school degree. However, maternal socioeconomic status is only a proxy of maternal IQ and the use of socioeconomic determinants does not exclude the possibility of residual confounding by maternal cognition. To the best of our knowledge, no previous study has shown that maternal intelligence can further explain season of birth variations in childhood IQ.

The biological explanations that were suggested to underlie the seasonal pattern in neurodevelopment explained a relatively small portion of the association between child season of birth and non-verbal IQ in our study. We did not observe a meaningful effect of maternal nutritional intake, vitamin D status or maternal exposure to fever during pregnancy on the association between child season of birth and IQ. Nutrition is regarded as an unlikely driver of seasonal birth outcomes in developed countries, where food supply fluctuates little over the year. ${ }^{27}$ Likewise, maternal and cord blood vitamin $\mathrm{D}$ concentrations had only a very modest improvement of the model. Previous studies of the association between maternal infections during pregnancy and neurodevelopmental outcomes such as schizophrenia varied in design. Findings are largely inconclusive and, if anything, explain little of the seasonal variation in mental health problems. ${ }^{28} 29$

Similarly, the relative age difference of children in the same class explained little of the seasonal pattern in IQ at age 6 years. This most likely reflects the schooling pattern of the Netherlands strictly by age with a staged school entry. Moreover, the initial age adjustment (we observed a slightly higher IQ in younger children) probably also accounted for age differences in the class. In contrast, Lawlor et al in the Aberdeen Children of the 1950s cohort showed that the seasonal pattern in agestandardised intelligence scores was largely explained by relative age. ${ }^{30}$ This effect in the 1950 s study may well be a birth cohort phenomenon or more relevant to other countries than the Netherlands. The current practice in the Netherlands is to school children strictly by age without any formal schooling criteria. Thus, we were able to correct adequately for the relative age as compared with peers in the classroom, yet observed a much less effect of age at schooling entry on IQ than in the Aberdeen Children of the 1950s cohort. Age at school entrance was not taken into account, as age variation at school entrance is very limited in the current population. In the Netherlands, public education law makes attendance in preschool compulsory by the age of 5 years. Furthermore, $98 \%$ of the Dutch children attend preschool from their fourth birthday onwards. ${ }^{13}$

Covariations with season of birth have been reported to the aetiology of many aspects of somatic and mental disorders, development and social adaptations varying from left-handedness ${ }^{31}$ and shyness ${ }^{32}$ to diabetes ${ }^{33}$ and epilepsy. ${ }^{34}$ Our observations emphasise that factors considered as instrumental variables, such as season of birth, may yet be heterogeneous within the general population and therefore likely to generate type I errors. Since almost all of these outcomes in these studies are known to vary by socioeconomic status, it is most likely that many of these apparent associations resulted from confounding rather than any true biological effect.

With the inclusion of intermediate variables in the model, in principle we assumed that there is no unmeasured confounding for the intermediate variables and the outcome. If an unknown variable confounds the association of the mediating variable with the outcome, bias may occur. Depending on the direction and magnitude, bias by incomplete adjustment may lead to either an overestimation or underestimation of the indirect 
effect on the outcome. Most likely, the effect of an unmeasured confounder with the outcome is in the same direction of the intermediate variable which would lead to an overestimation of the direct effect. Moreover, we ordered the models so that important confounders such as maternal sociodemographic characteristics are included before intermediates such as fever and CRP levels are tested, and thus these associations are automatically controlled for this covariate.

Our study has notable strengths, including the large sample size and prospective data assessments; however, some limitations need to be considered. First, although the study relied on the presence of multiple variables to explain season of birth effects, in particular prenatal factors may not have been assessed with enough precision. Some variables, for example, maternal exposure to fever, were based on questionnaires that entail the risk of under-reporting or over-reporting, while our laboratory measurements relied on single measurements only. More observational data or repeated measurements could have increased precision. Moreover, certain variables have missing values of over $20 \%$, for example, maternal nutrition and vitamin D status. Both limitations could lead to misclassification of the covariates. If such misclassification occurred, this most likely decreased the variance that we were able to explain for variables both on and not on the causal pathway.

Second, we found differences of just over one point on the IQ scale. It is unlikely that such a difference exerts an effect on performance in everyday life such as school achievement on an individual level. Nevertheless, owing to the large number of potentially affected children, at population level the impact on IQ is of interest. $^{35}{ }^{36}$ Furthermore, a relatively small effect size on intelligence was expected and compared well with previous studies attempting to explain the seasonal birth patterns of $\mathrm{IQ}^{37}$ Intelligence is a highly complex phenotype which is the net result of a wide range of biological processes and any variable such as season of birth is unlikely to signal a strong effect.

Third, another limitation is that higher educated mothers and Dutch children were more likely to be included in the study, which resulted in an underrepresentation of more disadvantaged families. This selective participation and loss to follow-up would lead to biased effect estimates if associations of season of birth with child non-verbal IQ would be different between those mothers included and not included in the analyses. This seems unlikely as we observed no consistent differences in distribution of seasonality of birth between children participating and not participating in follow-up.

Finally, the relative impact of prenatal and postnatal factors will depend on environmental conditions which may differ per population. The generalisability of our findings to other populations may therefore be limited.

In this study, all covariates assessed explained $65 \%$ of the effect estimate of the association of child season of birth and IQ at age 6 years. This is quite considerable compared with other studies using social and psychological factors to explain IQ.

There are several ways to model the seasonal variation. Previous literature relating season of birth to health outcomes primarily focused on astronomic and meteorological seasons. Astronomical seasons are based on the position of the earth in relation to the sun, whereas meteorological seasons are created by breaking the seasons down into groupings of 3 months based on the annual temperature cycle as well as our calendar. When we used the meteorological seasons as a risk indicator, the association with IQ was slightly weaker. If anything, we would have expected the opposite if biological factors underlie the seasonal IQ patterns. Lawlor $e t a l^{30}$ are one of the few researchers who fitted an adapted cosinor curve to the seasonal rhythm. However, the association of seasonal patterns of birth with IQ does not necessarily adhere to such periodic cycles but instead was better modelled in this study with seasonal patterns.

Our results might suggest that season of birth is a weak but significant indicator of numerous underlying processes related to child IQ. Sociodemographic factors, related to planning of pregnancy, and not biological confounders are of the highest importance in this association. Women giving birth in spring may differ from women giving birth in winter in ways that are related to the outcome of interest. Our observations emphasise that factors considered instrumental variables, such as season of birth, may yet be heterogeneous within the general population and therefore likely to reflect spurious associations.

\section{Author affiliations}

${ }^{1}$ The Generation R Study Group, Erasmus MC, University Medical Center, Rotterdam, The Netherlands

${ }^{2}$ Department of Child and Adolescent Psychiatry/Psychology, Erasmus MC, University Medical Center, Rotterdam, The Netherlands

${ }^{3}$ Department of Obstetrics and Gynecology, Erasmus MC, University Medical Center, Rotterdam, The Netherlands

${ }^{4}$ Department of Pediatrics, Erasmus MC, University Medical Center, Rotterdam, The Netherlands

${ }^{5}$ Department of Epidemiology, Erasmus MC, University Medical Center, Rotterdam, The Netherlands

${ }^{6}$ Department of Psychiatry, Erasmus MC, University Medical Center, Rotterdam, The Netherlands

Acknowledgements The Generation R Study is conducted by the Erasmus Medical Center in close collaboration with the School of Law and the Faculty of Social Sciences at the Erasmus University, Rotterdam, the Municipal Health Service, Rotterdam area, and the Stichting Trombosedienst and

Artsenlaboratorium Rijnmond (Star-MDC), Rotterdam. The authors gratefully acknowledge the contribution of participating mothers, general practitioners, hospitals, midwives and pharmacies in Rotterdam, the Netherlands.

Contributors NHG-vM performed the statistical analysis and drafted the manuscript. RPMS-T, VWVJ, AH, FCV and HT substantially contributed to the design of the study and the analysis and interpretation of the data; approved of its final version; and critically revised the manuscript for important intellectual content.

Funding This work was supported by the Erasmus Medical Center, Erasmus University Rotterdam, the Netherlands Organization for Health Research and Development (ZonMw 'Geestkracht' program (grant number 1.00.1003)), 
the Netherlands Organisation for Scientific Research, the Ministry of Health, Welfare and Sport, and the Ministry of Youth and Families and the Sophia Foundation for Medical Research (grant number 268 to NGM).

Competing interests None declared.

Ethics approval The study has been approved by the Medical Ethics Committee of the Erasmus Medical Center, University Medical Centre, Rotterdam.

Provenance and peer review Not commissioned; externally peer reviewed.

Data sharing statement No additional data are available.

Open Access This is an Open Access article distributed in accordance with the Creative Commons Attribution Non Commercial (CC BY-NC 4.0) license, which permits others to distribute, remix, adapt, build upon this work noncommercially, and license their derivative works on different terms, provided the original work is properly cited and the use is non-commercial. See: http:// creativecommons.org/licenses/by-nc/4.0/

\section{REFERENCES}

1. Torrey EF, Miller J, Rawlings R, et al. Seasonality of births in schizophrenia and bipolar disorder: a review of the literature. Schizophr Res 1997;28:1-38.

2. McGrath JJ, Saha S, Lieberman DE, et al. Season of birth is associated with anthropometric and neurocognitive outcomes during infancy and childhood in a general population birth cohort. Schizophr Res 2006;81:91-100.

3. Brown AS, Vinogradov S, Kremen WS, et al. Prenatal exposure to maternal infection and executive dysfunction in adult schizophrenia. Am J Psychiatry 2009;166:683-90.

4. Brown AS, Susser ES, Butler PD, et al. Neurobiological plausibility of prenatal nutritional deprivation as a risk factor for schizophrenia. J Nerv Ment Dis 1996;184:71-85.

5. Bhutta AT, Cleves MA, Casey PH, et al. Cognitive and behavioral outcomes of school-aged children who were born preterm: a meta-analysis. JAMA 2002;288:728-37.

6. Eriksen W, Sundet JM, Tambs K. Birth weight standardized to gestational age and intelligence in young adulthood: a register-based birth cohort study of male siblings. Am J Epidemiol 2010;172:530-6.

7. Currie J, Schwandt $\mathrm{H}$. Within-mother analysis of seasonal patterns in health at birth. Proc Natl Acad Sci USA 2013;110:12265-70.

8. McGrath J. Hypothesis: is low prenatal vitamin D a risk-modifying factor for schizophrenia? Schizophr Res 1999;40:173-7.

9. Goodman R, Gledhill J, Ford T. Child psychiatric disorder and relative age within school year: cross sectional survey of large population sample. BMJ 2003;327:472.

10. Gledhill J, Ford T, Goodman R. Does season of birth matter? The relationship between age within the school year (season of birth) and educational difficulties among a representative general population sample of children and adolescents (aged 5-15) in Great Britain. Res Educ 2002;68:41-7.

11. Roenneberg T, Aschoff J. Annual rhythm of human reproduction I. Biology, sociology, or both?. J Biol Rhythms 1990;5:195-216.

12. Kruithof CJ, Kooijman MN, van Duijn CM, et al. The Generation R Study: Biobank update 2015. Eur J Epidemiol 2014;29:911-27.

13. CBS, Netherlands Statistics. Report Jeugd 2003, cijfers en feiten.

14. Tellegen PJ, Winkel M, Wijnberg-Williams B, et al. SON-R 2,5-7: The Snijders-Oomen nonverbal intelligence tests. Manual and research report. Amsterdam, The Netherlands: Hogrefe, 2005.

15. Prieler J. Raven's advanced progressive matrices. Austria: Schufried, 2003.
16. Ghassabian A, Steenweg-de Graaff J, Peeters RP, et al. Maternal urinary iodine concentration in pregnancy and children's cognition: results from a population-based birth cohort in an iodine-sufficient area. BMJ Open 2014;4:e005520.

17. Verburg BO, Steegers EA, De Ridder M, et al. New charts for ultrasound dating of pregnancy and assessment of fetal growth: longitudinal data from a population-based cohort study. Ultrasound Obstet Gynecol 2008;31:388-96.

18. Eyles DW, Feron F, Cui X, et al. Developmental vitamin D deficiency causes abnormal brain development. Psychoneuroendocrinology 2009;34(Suppl 1):S247-57

19. Jaddoe VW, Bakker R, van Duijn CM, et al. The Generation R Study Biobank: a resource for epidemiological studies in children and their parents. Eur J Epidemiol 2007;22:917-23.

20. Klipstein-Grobusch K, den Breeijen JH, Goldbohm RA, et al. Dietary assessment in the elderly: validation of a semiquantitative food frequency questionnaire. Eur J Clin Nutr 1998;52:588-96.

21. Donders-Engelen M, Heijden van der L, Hulshof KF. Maten, Gewichten en codenummers, Human Nutrition of TNO and Wageningen University, Wageningen. 2003.

22. Netherlands Nutrition Centre. Nevo: Dutch food composition database 2006. The Hague: Netherlands Nutrition Centre, 2006.

23. Watson PE, McDonald BW. Seasonal variation of nutrient intake in pregnancy: effects on infant measures and possible influence on diseases related to season of birth. Eur J Clin Nutr 2007;61:1271-80.

24. Basso O, Olsen J, Bisanti L, et al. Are seasonal preferences in pregnancy planning a source of bias in studies of seasonal variation in reproductive outcomes? The European Study Group on Infertility and Subfecundity. Epidemiology 1995;6:520-4.

25. Haandrikman K. Seizoensfluctuaties in geboorten: veranderende patronen door planning? Bevolkingstrends 2004;52:14-22.

26. Buckles KS, Hungerman DM. Season of birth and later outcomes: old questions, new answers. Rev Econ Stat 2013;95:711-24.

27. Lee SJ, Steer PJ, Filippi V. Seasonal patterns and preterm birth: a systematic review of the literature and an analysis in a London-based cohort. BJOG 2006;113:1280-8.

28. Zerbo O, losif AM, Walker C, et al. Is maternal influenza or fever during pregnancy associated with autism or developmental delays? Results from the CHARGE (CHildhood Autism Risks from Genetics and Environment) study. J Autism Dev Disord 2013;43:25-33.

29. Barr CE, Mednick SA, Munk-Jorgensen P. Exposure to influenza epidemics during gestation and adult schizophrenia. A 40-year study. Arch Gen Psychiatry 1990;47:869-74.

30. Lawlor DA, Clark H, Ronalds G, et al. Season of birth and childhood intelligence: findings from the Aberdeen Children of the 1950s cohort study. Br J Educ Psychol 2006;76(Pt 3):481-99.

31. Martin M, Jones GV. Handedness and season of birth: a gender-invariant relation. Cortex 1999;35:123-8.

32. Gortmaker SL, Kagan J, Caspi A, et al. Daylength during pregnancy and shyness in children: results from northern and southern hemispheres. Dev Psychobiol 1997;31:107-14.

33. Samuelsson U, Ludvigsson J. Seasonal variation of birth month and breastfeeding in children with diabetes mellitus. J Pediatr Endocrinol Metab 2001;14:43-6.

34. Procopio M, Marriott PK. Is the decline in diagnoses of schizophrenia caused by the disappearance of a seasonal aetiological agent? An epidemiological study in England and Wales. Psychol Med 1998;28:367-73.

35. Salkever DS. Updated estimates of earnings benefits from reduced exposure of children to environmental lead. Environ Res 1995;70:1-6.

36. Sternberg RJ, Grigorenko E, Bundy DA. "The predictive value of IQ". Merrill Palmer Q 2001;47:1-41.

37. Wallingford EL, Prout HT. The relationship of season of birth and special education referral. Psychol Schools 2000;37:379-87. 